\title{
BMJ Open Cerebrospinal fluid biomarkers for the diagnosis and prognosis of Parkinson's disease: protocol for a systematic review and individual participant data meta- analysis
}

\author{
Paolo Eusebi, ${ }^{1}$ Oskar Hansson, ${ }^{2,3}$ Silvia Paciotti, ${ }^{1}$ Massimiliano Orso, ${ }^{4}$ \\ Davide Chiasserini, ${ }^{1,5}$ Paolo Calabresi, ${ }^{1,6}$ Kaj Blennow, ${ }^{7}$ Lucilla Parnetti ${ }^{1}$
}

To cite: Eusebi P, Hansson 0, Paciotti S, et al. Cerebrospinal fluid biomarkers for the diagnosis and prognosis of Parkinson's disease: protocol for a systematic review and individual participant data meta-analysis. BMJ Open 2017;7:e018177. doi:10.1136/ bmjopen-2017-018177

- Prepublication history and additional material for this paper are available online. To view these files, please visit the journal online (http://dx.doi. org/10.1136/bmjopen-2017018177).

Received 13 June 2017 Revised 4 October 2017 Accepted 23 October 2017

CrossMark

For numbered affiliations see end of article.

Correspondence to

Paolo Eusebi;

paoloeusebi@gmail.com

\section{ABSTRACT}

Introduction Idiopathic Parkinson's disease (PD) is a progressive neurodegenerative disorder related to $\alpha$-synuclein misfolding and aggregation. For this reason, it belongs to the family of 'synucleinopathies', which also includes some other neurological diseases. Although imaging and ancillary investigations may be helpful in the diagnostic workup, the diagnosis of PD mostly relies on the clinician's expertise. Furthermore, there is a need today for markers that can track the disease progression in PD that might improve the evaluation of novel disease-modifying therapies. The cerebrospinal fluid (CSF) has been widely investigated with the purpose of finding useful diagnostic and prognostic biomarkers for PD.

Methods and analysis This systematic review protocol has been developed according to the Preferred Reporting Items for Systematic reviews and MetaAnalyses Protocol 2015 statement and was registered on the PROSPERO international prospective register of systematic reviews. An international collaboration will be established. We will search the Cochrane Library, Web of Science, Medline and Embase from inception, using appropriate search strategies. Individual participant data from all included studies will be merged into a single database. We will include any study assessing the diagnostic and prognostic role of CSF biomarkers in PD. To evaluate the risk of bias and applicability of primary diagnostic accuracy studies, we will use Quality Assessment of Diagnostic Accuracy Studies-2 and Quality in Prognostic Studies. We will use standard meta-analytic procedures. We will first explore the utility of each CSF biomarker in turn. For each biomarker, we will assess its diagnostic and prognostic utility by means of receiver operating characteristic analysis and regression models. We will then move towards a multivariate approach considering different panels of biomarkers.

Ethics and dissemination Our study will not include confidential data, and no intervention will be involved, so ethical approval is not required. The results of the study will be reported in international peer-reviewed journals.
Strengths and limitations of this study

- The planned systematic review and individual participant data (IPD) meta-analysis aim to comprehensively assess the evidence regarding the diagnostic and prognostic utility of cerebrospinal fluid (CSF) biomarkers in Parkinson's disease.

- The planned systematic review and meta-analysis will be the first summary of the evidence in the field with an IPD approach.

- Heterogeneity in the design and conduct of the primary studies could make it difficult to reach exhaustive conclusions.

- We also expect that, due to interlaboratory variation and different producers/vendors of kits/platforms for measuring CSF biomarkers, it will be difficult to have defined and validated cut-off for each marker.

\section{BACKGROUND}

Idiopathic Parkinson's disease (PD) is a progressive neurodegenerative disorder related to $\alpha$-synuclein misfolding and aggregation. For this reason, it belongs to the family of 'synucleinopathies', which also includes some other neurological diseases, such as multiple system atrophy (MSA) and dementia with Lewy bodies. Clinical diagnosis of PD is based on the presence of bradykinesia accompanied by at least one other characteristic, such as tremor, rigidity and impaired postural reflexes. ${ }^{1}$ Typically, patients suffering from PD show a good response to symptomatic treatment with dopaminergic therapies. However, in addition to PD, several disorders cause parkinsonism, including atypical parkinsonian disorders such as progressive supranuclear palsy, corticobasal degeneration and MSA, and the different parkinsonian disorders are often difficult to distinguish from each other, 
especially during the early clinical stages. ${ }^{2}{ }^{3}$ Although imaging and ancillary investigations may be helpful in the diagnostic workup ${ }^{4-6}$ the accurate diagnosis of PD mostly relies on the clinician's expertise and consequently objective diagnostic methods are needed. Furthermore, there is a need today for markers that can track the disease progression in $\mathrm{PD}$, which might improve the evaluation of novel disease-modifying therapies. In the last decades, an increasing number of studies have been performed with the purpose of finding useful diagnostic and prognostic biomarkers for PD.

The cerebrospinal fluid (CSF) has been widely investigated as a source of reliable biomarkers for neurodegenerative diseases, especially for Alzheimer's disease but also for PD and atypical parkinsonian disorders, because CSF is the biological fluid closest to the brain. Unlike plasma, CSF is not separated from the brain by the blood-brain barrier. Hence, proteins linked to brain-specific activities or to disease processes are more represented in the CSF than in other fluids or tissues. The investigation of CSF biomarkers for PD diagnosis began in the 60s. Many CSF proteins were tested as biomarkers for the diagnosis of PD. Several have received attention and have been listed (with a non-exhaustive purpose, see Magdalinou et $a l^{1}$ ): total, oligomeric and phosphorylated $\alpha$-synuclein; $A \beta-42$; total and phosphorylated tau; neurofilament light-chain protein; oxidative stress markers (DJ-1, 8-OHdG and urate); and lysosomal dysfunction ( $\beta$-glucocerebrosidase).

\section{Rationale for a systematic review and an individual participant data meta-analysis}

We propose a systematic review and an individual participant data (IPD) meta-analysis for the evaluation of the diagnostic and prognostic utility of CSF biomarkers in PD. Despite the increasing number of published studies, few systematic reviews with meta-analysis were identified. ${ }^{7-10}$ All the evidence synthesis is about the diagnostic role of synuclein species in PD and other parkinsonisms. No evidence exists regarding the role of other CSF markers or for evaluating prognostic accuracy.

IPD represents the gold standard of meta-analysis and offers several advantages that could be of primary interest in the field of CSF biomarkers in $\mathrm{PD}^{11}$ : an increase in the total sample size, thus reducing the risk of false-positive findings and increasing the precision of study results; an increase in the case mix variability and the potential generalisability of prediction models across subgroups, settings and countries; the standardisation of the statistical analysis methods across IPD sets; more complex associations can be investigated, such as non-linearity of predictor effects, predictor interactions and time-varying predictor effects.

\section{Research aim}

This systematic review will consider studies investigating the value of CSF biomarkers in PD and will aim to determine whether the CSF biomarkers (alone or in combination) have the potential to:
- distinguish PD from healthy subjects, neurological controls and other atypical parkinsonisms;

- track disease progression as measured by dependence, motor impairment and cognitive decline;

- predict unfavourable outcomes such as dependence, motor impairment and cognitive decline.

\section{METHODS}

The IPD meta-analysis will be performed following the methods recommended by the IPD meta-analysis methods group of the Cochrane collaboration (http:// ipdmamg.cochrane.org/resources) and other published guidelines. ${ }^{12}$ This research protocol has been developed according to the Preferred Reporting Items for Systematic reviews and Meta-Analyses Protocol (PRISMA-P) 2015 statement and was registered on the PROSPERO Database (CRD42017052688). The actual start date of the study is 1 July 2017 and the anticipated completion date is 1 March 2019.

\section{Selection criteria}

Types of study

The following research and study designs will be considered: cross-sectional, prospective, longitudinal, randomised controlled trials, quasi-randomised clinical trials or controlled clinical trials.

\section{Types of participants}

Studies that included patients with PD will be considered. For testing the diagnostic accuracy of CSF biomarkers, we will require the presence of another group of individuals who could be either a group of neurological/healthy controls and/or patients with atypical parkinsonism. Studies with mixed populations will be included as long as data for relevant patients are extractable. CSF measurements must be centralised and blinded to diagnosis.

Setting

Studies in any setting will be included.

\section{Diagnostic criteria}

Movement Disorder Society Clinical Diagnostic Criteria for Parkinson's Disease, UK Parkinson's Disease Society Brain Bank criteria or the National Institute of Neurological Disorders and Stroke.

\section{Clinical outcomes}

\section{Primary outcomes:}

- Diagnostic accuracy of CSF biomarkers, alone or in combination, in distinguishing PD from controls or other parkinsonisms.

- Accuracy in predicting/tracking:

- disease staging as measured by modified Hoehn and Yahr (H\&Y) Scale;

- dependence/disability as measured by Modified Schwab and England activities of daily living (ADL), Barthel ADL Index and ADL/instrumental activities of daily living (IADL) scales; 
- progression of motor symptoms by means of Unified Parkinson's Disease Rating Scale (UPDRS) part III;

- cognitive impairment as measured by changes in Montreal Cognitive Assessment (MoCA) or MiniMental State Examination (MMSE) scores;

- progression to mild cognitive impairment and/or dementia.

Secondary outcomes: progression in terms of other measures of dependence/disability, motor and cognitive functions.

\section{Language}

No language restrictions will be applied.

\section{Search strategy}

Bibliographic databases (Medline, Embase, ISI Web of Science and The Cochrane Library) will be searched from inception to date. Searches will use combinations of index and text words encompassing: (a) patients with PD and (b) CSF. Publicly available registers will be searched for ongoing studies. In addition, abstracts of proceedings from biomarkers and neurodegenerative diseases conferences (national and international) will be consulted from 2011 onwards in order to capture studies that are not yet fully published. We will also search Google Scholar and OpenGrey in order to find additional papers of interest in the grey literature. To achieve a highly sensitive search, we will examine the first 300 hits of Google Scholar, as recommended by Haddaway and colleagues. ${ }^{13}$ An outline of the search strategy is available in the online Supplementary file 1 .

\section{Inclusion of studies}

\section{Study selection}

The process will be performed in two steps. In the first step, the titles (and abstracts if available) will be selected by two reviewers, using prespecified screening criteria related to whether the studies are about the assessment of CSF biomarkers in PD. Full texts of any potentially relevant articles will then be obtained and two reviewers will independently apply the full inclusion criteria. Any discrepancy between reviewers will be resolved by discussion or by referral to a third reviewer. Appropriate portions of non-English language articles will be translated where necessary.

\section{Invitations to join collaborative work}

Authors of selected studies will be invited to join the collaboration by providing us with an IPD. Contact information will be identified from the published studies or an online search. We will contact the main (corresponding) authors and provide them with the IPD meta-analysis protocol and a cover letter explaining the main purposes of the study. If we receive no response from the corresponding author, other investigators from the study will be contacted following a contribution order (equally contributed, senior author and others). We will search the grey literature extensively to check for publication bias. We will contact all authors of relevant unpublished studies with online search and request data.

\section{Quality assessment}

Two reviewers will assess quality of the included studies independently in accordance both with Quality Assessment of Diagnostic Accuracy Studies-2 (QUADAS-2) guidelines for diagnostic test studies ${ }^{14}$ and with Quality in Prognostic Studies (QUIPS) tool. ${ }^{15}$ We will perform a pilot QUADAS-2 and QUIPS assessment on two papers. If agreement is poor, we will refine the signalling questions. We will not use QUADAS-2 and QUIPS assessment to provide a summary quality score. We will produce a narrative summary describing numbers of studies that we considered at high/low/unclear (QUADAS-2) or high/ moderate/low (QUIPS) risk of bias as well as concerns regarding applicability.

\section{Development of the database \\ Data collection}

IPD from all included studies will be merged into a single database. We will request the data for all patients. A minimum set of data items is required in order to accept the study data, which need to include the following information: the H\&Y stage, UPDRS-III assessment and at least one neuropsychological assessment among MMSE and MoCA. We will provide authors with a list of required data items (see the online supplementary materials for the variable list), but we will accept data in all formats in order to minimise the amount of work for study authors and to ensure maximum participation. We will provide a data dictionary in order to maximise clarity of requests. The study authors can transfer raw data with a variety of secure methods depending on their preferences (we will, however, provide a guide for transferring files via email with password-protected archives). A data transfer agreement will be signed in order to: prohibit attempts to reidentify or contact study participants; address any requirements regarding planned outputs of proposed research; prohibit non-approved uses or further distribution of the data; and ensure that data has been collected in a study approved by a local ethics committee.

\section{Data checking}

Two independent researchers will check the quality of the collected data. For each variable, we will calculate the proportion of missing values and we will compare this with their reporting in the original publication. Descriptive statistics will be calculated for all the variables in each dataset to compare the values with the ones tabulated in the original publication. Discrepancies will be discussed and solved with the study authors.

\section{Data merging}

Raw datasets will be saved in their original formats and then converted to a common format by renaming and labelling the variables for each study in a consistent manner. Datasets will be combined to form a new master dataset, including a variable indicating the original study. 


\section{Confidentiality, data storage and handling}

The anonymised (ie, de-identified) dataset supplied by each collaborator and the final datasets will be used only for the purposes stated in this protocol and analyses set out in the analysis plan. All datasets will be stored in password-protected files on a secure computer accessible only by members of the management group (see below), and archived in accordance with patient data archiving procedures required by the Italian National Health Service. The datasets will not be shared with anyone outside the collaborative group without the express permission of each collaborator.

\section{Data analysis}

\section{IPD meta-analysis}

We will use standard meta-analytic procedures incorporating all available IPD. We will first explore the utility of each CSF biomarker in turn. For each biomarker we will perform receiver operating characteristic analysis and regression models in order to assess its diagnostic and prognostic utility. We will then move towards a multivariate approach considering different panels of biomarkers. Missing data will be handled according to multiple imputation techniques. Study heterogeneity will be investigated and reported. We will fully account for heterogeneity by performing random effects models. ${ }^{12} 16$

\section{Inclusion of aggregate data}

For studies that do not provide an IPD, we will undertake additional analyses in which we combine IPD when they are available with aggregate data when they are not.

\section{Sensitivity analysis}

We plan to conduct a sensitivity analysis by restricting the analysis to studies classified as being of good quality.

\section{Checking for publication and data availability bias}

We will examine funnel plots to investigate association between study size and effect size (which could be due to publication bias), with and without studies lacking IPD. We will describe study-level and patient-level characteristics of included studies. We will report the meta-analysis of IPD from studies that have supplied IPD, and a meta-analysis that combines the IPD with the aggregate data from the studies that will not provide IPD.

\section{Statistical analysis plan}

The analyses described above are the main analyses we propose to conduct and will constitute the core of the Statistical Analysis Plan (SAP), which will be detailed between receipt of the data and the full data analysis, in which we will specify what models and subgroup analyses will be performed. Changes and/or additional analyses may be indicated as the project progresses.

\section{Collaboration building}

The management group will be the core of the collaboration and its main purpose will be to effectively develop the activities of the larger collaborative group.
We have set up a management group encompassing the heterogeneous skills needed for the required work of the project. The management group includes persons with relevant expertise in key areas such as neurology, biochemistry, biostatistics, healthcare planning and evidence synthesis methodology. The management group will be responsible for contacting and cooperating with study authors, data collection, checking and analysis, interpretation of findings and drafting manuscripts for publication purposes.

The IPD meta-analysis collaborative group will include all members of the management group, clinicians with expertise in PD and a representative from each of the included studies. New collaborators will be invited as eligible studies are completed. Members of the collaborative group will be involved from the beginning of the study and given the opportunity to participate to the key steps, including the approval of the SAP and interpretation of the results.

\section{Publication policy}

All publications resulting from the study will be in the name of the collaborative group, with the contribution of members being described at the end of the paper. Manuscripts will be drafted by the management group and sent to all members of the collaborative group for comments prior to submission for peer-reviewed journals.

\section{Ethical issues}

All databases will include de-identified results, meaning that no clinical or biomarker data can be traced back to individual patients. Thus, the study does not require ethics approval. Moreover, in order to fulfil the requirements of the Italian national privacy authority, contributors have only to submit anonymised datasets (ie, the linking between original study id and meta-analysis id will be retained by the contributor and not shared, in any case, with the project team). The main study author will be allowed to specify any additional restrictions on data usage or storage (beyond those stated above) that they may wish to impose.

\section{DISCUSSION}

It is anticipated that the results of this project will represent a significant step towards informing clinical practice by indicating whether CSF biomarkers have diagnostic and/or prognostic utility.

\section{Author affiliations}

${ }^{1}$ Department of Medicine, Neurology Clinic, University of Perugia, Ospedale Santa Maria della Misericordia, Perugia, Perugia, Italy

2Department of Clinical Sciences, Clinical Memory Research Unit, Lund University, Lund, Sweden

${ }^{3}$ Memory Clinic, Skåne University Hospital, Malmö, Sweden

${ }^{4}$ Health Planning Service, Department of Epidemiology, Regional Health Authority of Umbria, Perugia, Italy

${ }^{5}$ Oncoproteomics Laboratory, VU University Medical Center, Amsterdam, The Netherlands

${ }^{6}$ Fondazione Santa Lucia, Rome, Italy

${ }^{7}$ Clinical Neurochemistry Laboratory, Institute of Neuroscience and Physiology,

Sahlgrenska Academy at University of Gothenburg, Sahlgrenska University Hospital, Mölndal, Sweden 
Contributors PE: conceived the idea, and planned and designed the study protocol. PE and LP: wrote the first draft. M0: designed the search strategy and planned the data extraction. PE: planned the data extraction and statistical analysis. PC, $\mathrm{OH}$ and KB: provided critical insights. All authors: approved and contributed to the final written manuscript.

Funding This project is funded by the Italian Ministry of Health (Grant ID: GR-2013-02357757).

Competing interests None declared.

Provenance and peer review Not commissioned; externally peer reviewed.

Open Access This is an Open Access article distributed in accordance with the Creative Commons Attribution Non Commercial (CC BY-NC 4.0) license, which permits others to distribute, remix, adapt, build upon this work non-commercially, and license their derivative works on different terms, provided the original work is properly cited and the use is non-commercial. See: http://creativecommons.org/ licenses/by-nc/4.0/

(c) Article author(s) (or their employer(s) unless otherwise stated in the text of the article) 2017. All rights reserved. No commercial use is permitted unless otherwise expressly granted.

\section{REFERENCES}

1. Magdalinou N, Lees AJ, Zetterberg H. Cerebrospinal fluid biomarkers in parkinsonian conditions: an update and future directions. J Neurol Neurosurg Psychiatry 2014;85:1065-75.

2. Hughes AJ, Daniel SE, Kilford L, et al. Accuracy of clinical diagnosis of idiopathic Parkinson's disease: a clinico-pathological study of 100 cases. J Neurol Neurosurg Psychiatry 1992;55:181-4.

3. Tolosa E, Wenning G, Poewe W. The diagnosis of Parkinson's disease. Lancet Neurol 2006;5:75-86.

4. Mahlknecht P, Hotter A, Hussl A, et al. Significance of MRI in diagnosis and differential diagnosis of Parkinson's disease. Neurodegener Dis 2010;7:300-18.
5. Vlaar AM, de Nijs T, Kessels AG, et al. Diagnostic value of 123l-ioflupane and 1231-iodobenzamide SPECT scans in 248 patients with parkinsonian syndromes. Eur Neuro/ 2008;59:258-66.

6. Hall S, Öhrfelt A, Constantinescu R, et al. Accuracy of a panel of 5 cerebrospinal fluid biomarkers in the differential diagnosis of patients with dementia and/or parkinsonian disorders. Arch Neurol 2012;69:1445-52.

7. Sako W, Murakami N, Izumi Y, et al. Neurofilament light chain level in cerebrospinal fluid can differentiate Parkinson's disease from atypical parkinsonism: Evidence from a meta-analysis. J Neurol Sci 2015;352:84-7.

8. Gao L, Tang H, Nie K, et al. Cerebrospinal fluid alpha-synuclein as a biomarker for Parkinson's disease diagnosis: a systematic review and meta-analysis. Int $J$ Neurosci 2015;125:645-54.

9. Blennow K, Biscetti L, Eusebi P, et al. Cerebrospinal fluid biomarkers in Alzheimer's and Parkinson's diseases-From pathophysiology to clinical practice. Mov Disord 2016;31:836-47.

10. Eusebi P, Giannandrea D, Biscetti L, et al. Diagnostic utility of cerebrospinal fluid $\alpha$-synuclein in Parkinson's disease: A systematic review and meta-analysis. Mov Disord 2017;32:1389-1400.

11. Debray TP, Riley RD, Rovers MM, et al. Individual participant data (IPD) meta-analyses of diagnostic and prognostic modeling studies: guidance on their use. PLOS Med 2015;12:e1001886.

12. Riley RD, Lambert PC, Abo-Zaid G. Meta-analysis of individual participant data: rationale, conduct, and reporting. $B M J$ 2010;340:c221.

13. Haddaway NR, Collins AM, Coughlin D, et al. The role of google scholar in evidence reviews and its applicability to grey literature searching. PLoS One 2015;10:e0138237.

14. Whiting PF, Rutjes AW, Westwood ME, et al. QUADAS-2: a revised tool for the quality assessment of diagnostic accuracy studies. Ann Intern Med 2011;155:529-36.

15. Hayden JA, Côté P, Bombardier C. Evaluation of the quality of prognosis studies in systematic reviews. Ann Intern Med 2006;144:427-37.

16. Michiels S, Baujat B, Mahé $\mathrm{C}$, et al. Random effects survival models gave a better understanding of heterogeneity in individual patient data meta-analyses. J Clin Epidemiol 2005;58:238-45. 